NASA Technical Paper 1118

Eigenvalue/Eigenvector Assignment

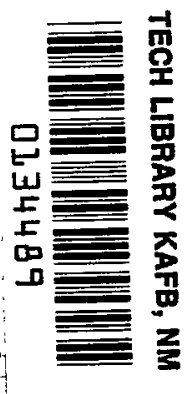

Using Output Feedback

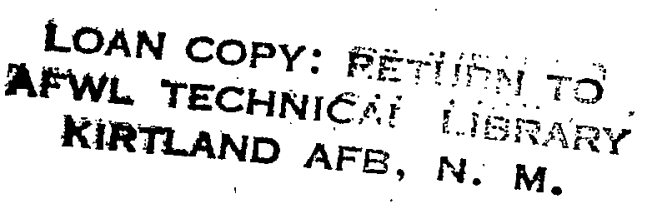

S. Srinathkumar

FEBRUARY 1978 
NASA Technical Paper 1118

\section{Eigenvalue/Eigenvector Assignment Using Output Feedback}

S. Srinathkumar

Langley Research Center

Hampton, Virginia

\section{N/Sก}

National Aeronautics and Space Administration

Scientific and Technical Information Office 


\section{SUMMARY}

The problem of eigenvalue assignment in a linear time-invariant system using output feedback is considered. New sufficient conditions are derived to assign an almost arbitrary set of minimum $(n, m+r-1)$ distinct eigenvalues where $\mathrm{n}$, $\mathrm{m}$, and $\mathrm{r}$ are the number of states, inputs, and outputs, respectively. These conditions precisely identify, the class of systems where such an assignment is impossible. The synthesis technique also highlights the freedom in selection of closed-loop eigenvectors under output feedback. The utility of eigenvalue/eigenvector assignment in transient response shaping is illustrated by the design of a controller for the lateral dynamics of an aircraft.

\section{INTRODUCTION}

Control system design based on eigenvalue or pole assignment has received a great deal of attention in the literature. It is well known that for a controllable system, if state variable feedback is employed, all eigenvalues can be assigned (ref. 1). Also it is known that for multi-input systems, the feedback law assigning a given set of eigenvalues is not unique and that different control laws can yield identical eigenvalues while yielding radically different eigenvectors. Since the eigenvectors determine the influence of each eigenvalue on each state variable response, failure to use the multi-input design freedom fully may result in undesirable mode coupling and other poor transient behavior. For n-state feedback systems, it has been shown (ref. 2) that with $m$ inputs, in addition to the assignment of all $\mathrm{n}$ eigenvalues, up to $\mathrm{m}$ entries in each eigenvector can be arbitrarily assigned. However, the problem of eigenvalue assignment using output feedback instead of state feedback has not yet been completely resolved. The problem of determining conditions under which all eigenvalues of a system can be arbitrarily assigned to a system under output feedback has been investigated in references 3 and 4 . Bounds on the number of states, in terms of number of inputs, outputs, controllability, and observability indices are established for complete pole assignability. Reference 5 shows that for a system with $r$ outputs, if $\mathrm{mr} \geq \mathrm{n}$ then the system is pole-assignable provided the feedback gain elements are allowed to be complex numbers. References 6 and 7 address the converse problem: given a controllable, observable system, how many eigenvalues can be arbitrarily assigned to the system. In general, it is concluded ( $r$ ef. 7 ) that minimum ( $n, m+r-1)$ eigenvalues can "almost" always be assigned to the system using output feedback. The qualification "almost" was introduced to cover classes of systems where such an assignment is impossible. In effect, the analysis in reference 7 does not precisely determine the conditions under which $(m+r-1)$ eigenvalues cannot be assigned to the system.

This report considers the problem of determining the number of eigenvalues assignable to a given system. By formulating an eigenvalue/eigenvector assignment problem, sufficient conditions required for the assignment of minimum $(n, m+r-1)$ eigenvalues are derived. These conditions precisely identify 
the class of systems which can be assigned only d eigenvalues, where maximum $(m, r) \leq d<(m+r-1)$. The new formulation permits the development of an algorithm to assign $(m+r-1)$ eigenvalues. In addition, $(r-1)$ eigenvectors can be partially assigned with, at most, $m$ entries in each vector arbitrarily chosen. In the event $n>(m+r-1)$, various synthesis alternatives to stabilize the system are also investigated since in this case all system eigenvalues cannot be assigned. The counter example of reference 7 is used to demonstrate the utility of the new sufficient conditions in identifying systems which cannot be assigned $(m+r-1)$ eigenvalues. Finally, the advantage of both eigenvalue and eigenvector assignments in response shaping is illustrated by designing a controller to meet the lateral handling qualities specifications for an aircraft.

\section{SYMBOLS}

Values are given in SI and U.S. Customary Units. Calculations were made in U.S. Customary Units.

A system matrix

$A \in R^{n \times n} n \times n$ real matrix $A$

ay lateral acceleration, $\mathrm{m} / \mathrm{sec}^{2}\left(\mathrm{ft} / \mathrm{sec}^{2}\right)$

$\mathrm{B} \quad$ input matrix

C measurement matrix

c measurement vector

$\bar{D} \quad$ matrix used in equation (45)

$\mathrm{D}(\mathrm{k})$ matrices defined by equation (B4)

$d, i, j, k, t \quad$ indices

$\mathbf{e}_{j}$ vector defined in notation (1) in appendix $B$

F matrix defined by equation (9)

$f_{j}(k-1)$ vector defined by step $3(a)$ of appendix $B$

G matrix defined by equation (10)

$\mathrm{g}_{\mathrm{j}}(\mathrm{k}) \quad$ vector defined by step $3(\mathrm{a})$ of appendix $\mathrm{B}$

$\mathrm{H}_{i} \quad$ matrices used in equation (23) and derived by substituting equation (6) into equation (7)

$h_{j}(k-1) \quad$ vector defined by step $3(a)$ of appendix $B$

Index (index) th order identity matrix 
$j \in\left\{\Delta^{(k)}\right\} \quad j$ is an element of index set $\Delta^{(k)}$

K feedback matrix

$\mathrm{L}_{i} \quad$ matrices used in equation (23) and derived by substituting equation (6) into equation (7)

l feedback vector defined by equation (17)

$M^{(k)}, M^{(k-1)}, M^{(1)}$ matrices defined by notation (6) in appendix $B$

m number of inputs

$\max \quad$ maximum value

min minimum value

N matrix defined by equation (13)

$\mathrm{n} \quad$ number of states

P matrix used in equation (46) and defined immediately afterward

p roll rate, $\mathrm{deg} / \mathrm{sec}$

$Q^{(k-1)}, Q^{(i)} \quad$ matrices defined by equation (B3)

$Q(0) \quad$ matrix defined by notation (3) in appendix $B$

q feedback vector defined by equation (18)

$r \quad$ number of outputs

$S \quad$ matrix defined by equation (8)

s vector defined immediately prior to equation (20)

$\mathrm{T}_{0}, \mathrm{~T}_{1}$ transformation matrices defined where used

$\mathrm{u} \quad$ input vector defined by equation (2)

$\mathrm{V}$ modal matrix (matrix of eigenvectors)

$v(r)$ matrix used in equation (12)

$\mathrm{v}, \mathrm{v}_{\mathbf{i}} \quad$ eigenvectors

w vector used in equation (6); a partition of $\mathrm{v}$

$\mathrm{x} \quad$ state vector defined by equations (1)

$\mathrm{x} \in \mathrm{R}^{\mathrm{n}} \mathrm{n} \times 1$ real vector $\mathrm{x}$ 


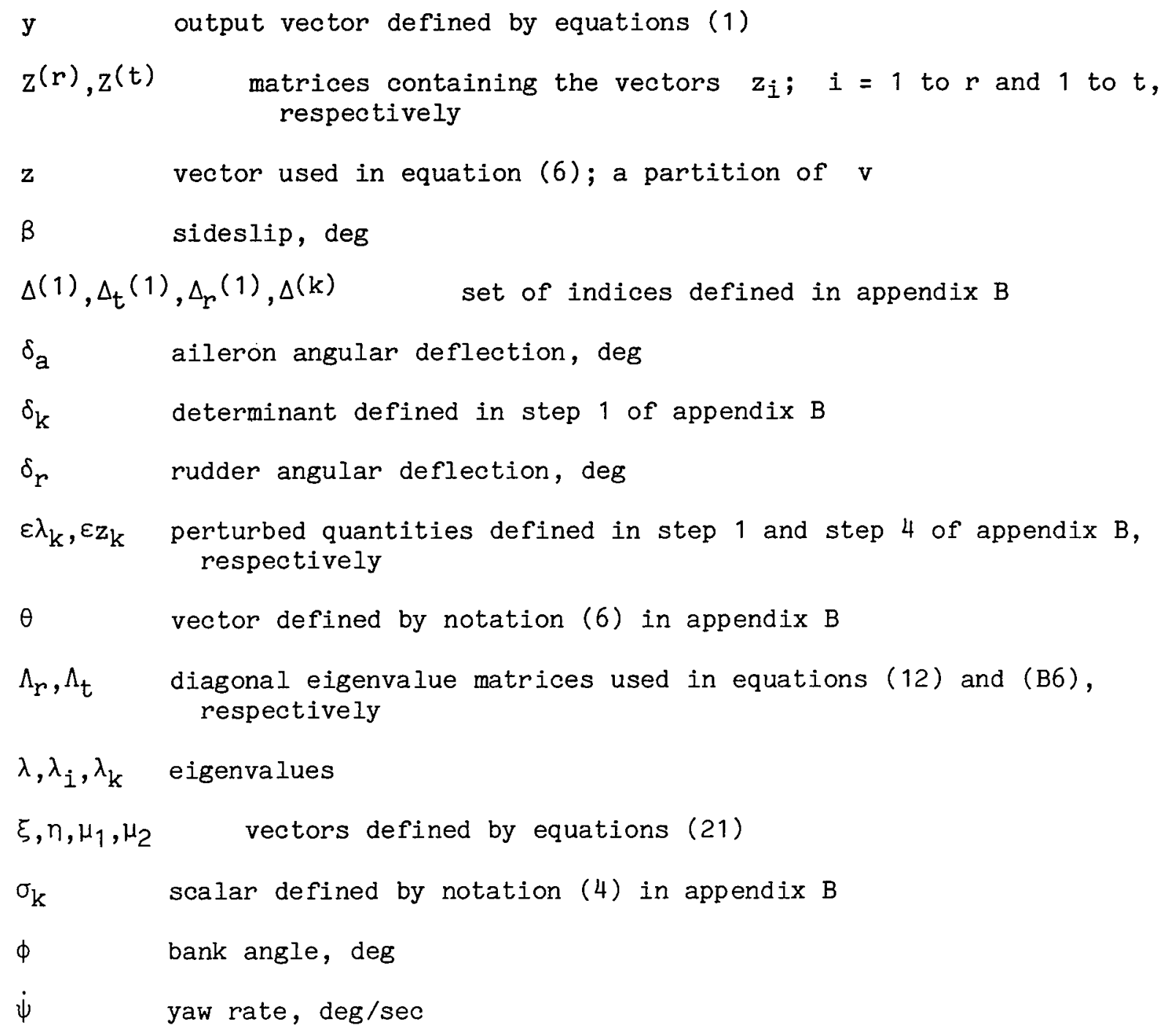

\section{EIGENVALUE/EIGENVECTOR ASSIGNMENT FORMULATION} system

Consider a linear, time invariant, multivariable, controllable, observable 


$$
\left.\begin{array}{l}
\dot{x}=A x+B u \\
y=C x
\end{array}\right\}
$$

where $x \in R^{n}, u \in R^{m}, y \in R^{r}$, and $B$ and $C$ are full rank; and for a nontrivial problem formulation, assume $\mathrm{m}, \mathrm{r}>1$ and $\mathrm{m}, \mathrm{r}<\mathrm{n}$. The problem is to find a control law of the form

$$
\mathrm{u}=\mathrm{Ky}
$$

in order to assign arbitrary eigenvalues for the closed-loop system. To indicate clearly the freedom available in the selection of closed-loop eigenvalues and eigenvectors under output feedback, the measurement matrix $C$ is assumed to be in a special canonical form:

$$
\begin{aligned}
& C=\left[C_{1}: C_{2}\right] \\
& C_{1}=\left[\begin{array}{c}
I_{t} \\
\cdot \\
0
\end{array}\right] \\
& C_{2}=\left[\begin{array}{l}
0 \\
\cdot \\
\dot{c}
\end{array}\right] \\
& c=\left[\begin{array}{lllll}
1 & 1 & . & . & 1
\end{array}\right]
\end{aligned}
$$

Here, $\quad t=r-1, \quad c_{1} \in R^{r \times t}, \quad c \in R^{1 \times n-t}$, and $I_{t}$ denotes a $t^{\text {th }}$ order identity matrix. Appendix A details a procedure for reducing any system $(C, A, B)$ to this special form.

The closed-loop system matrix $(A+B K C)$ after applying feedback law (2) satisfies

$$
(A+B K C) v_{i}=\lambda_{i} v_{i} \quad(i=1,2, \ldots, n)
$$

where $\lambda_{i}$ is the ith eigenvalue and $v_{i}$ is the corresponding eigenvector. The eigenvalue/eigenvector assignment problem is to determine the number of eigenvalues in equation (4) that can be arbitrarily assigned and to determine the freedom available in the selection of the associated eigenvectors.

In order to see what freedom exists in the choice of eigenvectors, write equation (4) in partitioned form as

$$
\left\{\left[\begin{array}{ccc}
A_{11} & \cdot & A_{12} \\
\cdot & \cdot & \cdot \\
A_{21} & \cdot & \cdot \\
A_{22}
\end{array}\right]+\left[\begin{array}{c}
B_{1} \\
\cdot \cdot \\
B_{2}
\end{array}\right] K C\right\} v_{i}=\lambda_{i} v_{i}
$$


where $A_{11}, B_{1} \in R^{m \times m}$ and $B_{1}$ is nonsingular. Since $B$ is full rank, the nonsingularity of $B_{1}$ can be assured, if necessary, by reordering the state variables in equations (1). Completing the multiplication of the partitioned matrices and some algebraic operations (ref. 8) permits equation (5) to be expressed as a set of constraints on the selection of eigenvectors. For clarity of presentation, these relations are detailed only for real eigenvalues. Extension to complex conjugate pairs in quasi-diagonal form yielding real eigenvector pairs is straightforward (ref. 8).

For real eigenvalues

$$
\begin{aligned}
& {\left[\lambda I_{n-m}-F\right] w=[G+\lambda S] z} \\
& {\left[A_{1}+B_{1} K C\right] v=\lambda z}
\end{aligned}
$$

where $\lambda$ is the eigenvalue, $v^{\prime}=\left[z^{\prime}: w^{\prime}\right] ; \quad v$ is the eigenvector with $z \in \mathrm{R}^{\mathrm{m}}$, and

$$
\begin{aligned}
& S=B_{2} B_{1}-1 \\
& F=A_{22}-S A_{12} \\
& G=A_{21}-S A_{11} \\
& A_{1}=\left[A_{11}: A_{12}\right]
\end{aligned}
$$

Equation (6) represents an underdetermined system of $n-m$ equations in $n$ unknowns. Thus $m$ eigenvector entries corresponding to the $z$-vector can be chosen arbitrarily provided $\lambda$ does not coincide with the spectrum of $F$. Examination of equation (7) reveals that at least $r$ eigenvalues and $r$ eigenvectors satisfying equation ( 6 ) can be assigned to the system in equations (1) by the feedback matrix

$$
\overline{\mathrm{K}}=\mathrm{B}_{1}-1\left[\mathrm{Z}(\mathrm{r}) \Lambda_{\mathrm{r}}-\mathrm{A}_{1} \mathrm{~V}^{(\mathrm{r})}\right][\mathrm{CV}(\mathrm{r})]^{-1}
$$

where $\Lambda_{r}$ is the diagonal matrix of $r$ eigenvalues and $Z^{(r)}$ and $V(r)$ have the form $T(r)=\left[t_{1}: t_{2}:\right.$...: $\left.t_{r}\right]$ (where $t_{i}$ are vectors). The solution to equation (12) is guaranteed provided the eigenvalues/eigenvectors are chosen to insure the nonsingularity of $[\mathrm{CV}(r)]$. It should be noted that in the case of state variable feedback $\left(C=I_{n}\right)$, all $n$ eigenvalues can be assigned to the system provided the modal matrix (matrix of eigenvectors) $\mathrm{V}=\left[\mathrm{v}_{1}: \mathrm{v}_{2}: \cdot \cdot \cdot \mathrm{v}_{\mathrm{n}}\right]$ is nonsingular. An algorithm which constructs such a nonsingular $\mathrm{V}$ is detailed in reference 8. Appendix B extends this algorithm to guarantee the nonsingularity of $[\mathrm{CV}(r)]$.

Notice that by carrying out this analysis on the dual system (B', $\left.A^{\prime}, C^{\prime}\right)$, it can be shown that $m$ eigenvalues can be assigned to the system. This analysis yields the following well-known result (ref. 9). 
Lemma 1: For system $(C, A, B), \max (m, r)$ eigenvalues can be assigned using output feedback.

The analysis so far indicates that only $\max (m, r)$ eigenvalues can be assigned to the system using output feedback. However, by sacrificing some degree of freedom in the selection of the associated eigenvectors it is possible to extend the number of eigenvalues that can be assigned to $\min (n, m+r-1)$ as is shown in the following section.

\section{ALGORITHM TO ASSIGN MINIMUM $(n, m+r-1)$ EIGENVALUES}

The basic approach in the development of this algorithm is to construct the output feedback law in equation (2) as a sum of two feedbacks $(\tilde{\mathrm{K}}+\hat{\mathrm{K}})$. The first feedback $(\tilde{K})$ assigns $t$ eigenvalues, and the second feedback assigns additional $\min (m, n-t)$ eigenvalues while ensuring the protection of the $t$ eigenvalues already assigned. The construction procedure yields a set of sufficient conditions for assigning $\min (n, m+r-1)$ eigenvalues. These conditions also help characterize the class of systems which cannot be assigned $\min (n, m+r-1)$ eigenvalues. Finally, some design freedom still exists to partially assign $(r-1)$ eigenvectors.

Step 1:

Assign $t$ eigenvalues and corresponding eigenvectors to the system in equations (1) and form the matrix

$$
\mathrm{N}=\left[\begin{array}{c}
\mathrm{N}_{1} \\
\cdot \overrightarrow{\mathrm{N}_{2}}
\end{array}\right]=\left[\mathrm{v}_{1}: \mathrm{v}_{2}: \cdot . \cdot \mathrm{v}_{\mathrm{t}}\right]
$$

with $N_{1} \in R^{t \times t}$ and nonsingular. Appendix $B$ details a procedure to construct $\mathrm{N}$. Let $\widetilde{\mathrm{K}}$ be the nonunique feedback (eq. (B6)) corresponding to this assignment. Then the closed-loop matrix is

$$
\widetilde{A}=A+B \widetilde{K} C
$$

Step 2:

Apply a coordinate transformation

$$
(\mathrm{C}, \tilde{\mathrm{A}}, \mathrm{B}) \rightarrow\left(\mathrm{CT}_{1}, \mathrm{~T}_{1}{ }^{-1} \tilde{\mathrm{A}} \mathrm{T}_{1}, \mathrm{~T}_{1}{ }^{-1} \mathrm{~B}\right)
$$

where

$$
\mathrm{T}_{1}=\left[\begin{array}{ccc}
\mathrm{N}_{1} & \cdot & 0 \\
\cdot & \cdot & \cdot \\
\mathrm{N}_{2} & \cdot & \cdot \\
\mathrm{I}_{\mathrm{n}-\mathrm{t}}
\end{array}\right]
$$


The transformed system has the form

$$
\left.\begin{array}{l}
\dot{\hat{x}}=\left[\begin{array}{ccc}
\Lambda_{t} & \cdot & \hat{A}_{12} \\
\cdot & \cdot & \cdot \\
0 & \cdot & \hat{A}_{22}
\end{array}\right] \hat{\mathrm{x}}+\left[\begin{array}{c}
\hat{\mathrm{B}}_{1} \\
\cdot \cdot \cdot \\
\hat{\mathrm{B}}_{2}
\end{array}\right] \mathrm{u} \\
\mathrm{y}=\left[\begin{array}{lll}
\hat{\mathrm{C}}_{1} & : & c_{2}
\end{array}\right] \hat{\mathrm{x}}
\end{array}\right\}
$$

where $\Lambda_{t} \in \mathrm{R}^{\mathrm{t} \times \mathrm{t}}$ is the diagonal matrix of eigenvalues assigned in step 1 . It should be noted that $C_{2}$ is invariant under the transformation $T_{1}$.

Step 3:

In order to assign additional eigenvalues to the system of equations (16) while protecting $t$ eigenvalues $\left(\Lambda_{t}\right)$ already assigned, the second feedback $(\hat{K})$ is restricted to be of unity rank of the form $\hat{\mathrm{K}}=\mathrm{q} l$ ' with $q \in \mathrm{R}^{\mathrm{m}}$, $l \in \mathrm{R}^{r}$, and $l$ is chosen so that

$$
\ell \cdot\left[\hat{c}_{1}: c_{2}\right]=\left[\begin{array}{lll}
0 & : c
\end{array}\right]
$$

Now $q$ must be chosen so that

$$
\left[\hat{\mathrm{A}}_{22}+\hat{\mathrm{B}}_{2 q \mathrm{c}}\right]
$$

is assigned $\min (m, n-t)$ eigenvalues.

Since $\left(\hat{A}_{22}, \hat{B}_{2}\right)$ is controllable, the following result holds:

Theorem 1: The single output subsystem $\left(c, \hat{A}_{22}, \hat{B}_{2}\right)$ can be assigned $\min (m, n-t)$ eigenvalues if and only if $(a)\left(c, \hat{A}_{22}\right)$ is observable and (b) $\hat{B}_{2}$ is full rank.

Theorem 1 follows directly from lemma 1. Further, conditions (a) and (b) restrict the admissible set of eigenvalue/eigenvector assignments in step 1. For conceptual convenience, these parametric restrictions are formulated in terms of controllability conditions of a fictitious dynamic system in the state variable representation.

After matrix operations in equations (14) and (16) have been performed, the submatrix $\hat{A}_{22}$ can be written as

$$
\hat{A}_{22}=\left[A_{22}+B_{2} \tilde{K} C_{2}\right]+\tilde{N}_{2}\left[A_{12}+B_{1} \tilde{K} C_{2}\right]
$$

where $\tilde{\mathrm{N}}_{2}=-\mathrm{N}_{2} \mathrm{~N}_{1}^{-1}$. Transposing equation (19) and writing $\tilde{\mathrm{K}} \mathrm{C}_{2}=\mathrm{sc}$, $\mathrm{s} \in \mathrm{R}^{\mathrm{m}}$ yields

$$
\hat{A}_{22}^{\prime}=A_{22}^{\prime}+c^{\prime} s^{\prime} B_{2}^{\prime}+A_{12}^{\prime} \tilde{N}_{2}^{\prime}+c^{\prime} s^{\prime} B_{1}^{\prime} \tilde{N}_{2}^{\prime}
$$


Equation (20) can now be looked upon as a system matrix derived by applying feedback to the dynamic system

$$
\left.\begin{array}{l}
\dot{\xi}=A_{22}^{\prime} \xi+A_{12}^{\prime} \mu_{1}+c^{\prime} \mu_{2} \\
n=B_{2}^{\prime} \xi+B_{1}^{\prime} \mu_{1}
\end{array}\right\}
$$

with $\xi \in \mathrm{R}^{\mathrm{n}-t}, \quad n \in \mathrm{R}^{\mathrm{m}}, \mu_{1} \in \mathrm{R}^{\mathrm{t}}, \mu_{2} \in \mathrm{R}^{1}, \mu_{1}=\tilde{\mathrm{N}}_{2}^{\prime} \xi, \mu_{2}=\mathrm{s}^{\prime} \mathrm{n}$, and $\hat{N}_{2}=-\mathrm{N}_{2} \mathrm{~N}_{1}{ }^{-1}$.

From equations (21) it can be shown that conditions (a) and (b) of theorem 1 are equivalent to the following conditions: (c) $\hat{B}_{2}=B_{2}+\tilde{N}_{2} B_{1}$ and is full

rank; (d) $\left\{\left[\mathrm{A}_{2} \dot{2}+\mathrm{A}_{1} \tilde{N}^{\prime}{ }_{2}^{\prime}\right], c^{\prime}\right\}$ is controllable. Conditions (c) and (d) clearly indicate the restriction on selection of the eigenvector parameters $\tilde{\mathrm{N}}_{2}$ in step 1. This yields the following sufficient conditions:

Theorem 2: The system ( $C, A, B)$ can be assigned min( $n, m+r-1)$ eigenvalues arbitrarily close to the desired set if the first $t$ eigenvalues and eigenvectors in step 1 are chosen so that

I $\quad \mathrm{N}_{1}$ is nonsingular

II $\hat{B}_{2}=\mathrm{B}_{2}+\tilde{\mathrm{N}}_{2} \mathrm{~B}_{1}$ and is full rank

III $\left\{\left[A_{2}^{\prime} 2+A_{12}^{\prime} \tilde{N}_{2}\right], c^{\prime}\right\}$ is controllable

"Arbitrarily close" in theorem 2 indicates that slight perturbations in eigenvalue specifications may be needed in the following situations:

(i) Assigned eigenvalues coincide with the spectrum of $F$ (appendix B).

(ii) An exact combination of eigenvalue/eigenvector specifications in step 1 may not yield a nonsingular $\mathrm{N}_{1}$ (appendix $\mathrm{B}$ ).

(iii) Coincident spectrum situation similar to situation (i) exists for the subsystem eigenvalue assignment of theorem 1.

Condition $I$ is required to guarantee the existence of the transformation $\mathrm{T}_{1}$ in step 2. This condition can be explicitly included in the synthesis procedure as detailed in appendix B. Condition III is obtained from the property that for system (eqs. (21)), the class of feedback from input $\mu_{1}$ should be restricted so that the controllability of the feedback system with respect to the input $\mu_{2}$ is preserved. Conditions II and III yield nonlinear algebraic constraints for the elements of the eigenvector parameter matrix $\widetilde{N}_{2}$ and thus, in general, can only be used as test conditions for each assignment in step 1. However, example 1 of the section entitled "Numerical Examples" shows how these conditions can be explicitly checked. 
Step 4:

If theorem 2 holds, $\min (m, n-t)$ eigenvalues can be assigned to the single output system $\left(c, \hat{A}_{22}, \hat{B}_{2}\right)$ by the feedback $q$ derived from lemma 1 .

Step 5:

The composite feedback law

$$
u=(\tilde{K}+\hat{K}) y
$$

assigns $\min (n, m+r-1)$ eigenvalues to the system.

\section{ASSIGNING $\mathrm{n}$ EIGENVALUES}

The development so far has revealed that for systems where $n>(m+r-1)$, all system eigenvalues cannot usually be assigned. However, by using equations (6) and (7), it is possible to derive conditions for assigning all eigenvalues of the system as follows.

If the eigenvalues to be assigned are noncoincident with the spectrum of F (if necessary, by a perturbation in specification), then equation (6) can be explicitly solved for the w-vector and substituted into equation (7). This substitution yields a set of homogeneous equations of the form

$$
\left[\mathrm{H}_{i}+\mathrm{B}_{1} \mathrm{KL}_{i}\right] \mathrm{z}_{i}=0 \quad(i=1,2, . . ., \mathrm{n})
$$

where $H_{i}$ and $L_{i}$ are readily derived. Then equation (23) has a nontrivial solution if and only if

$$
\text { rank }\left[\mathrm{H}_{i}+\mathrm{B}_{1} \mathrm{KL}_{i}\right]<\mathrm{m} \text { for all } i
$$

A set of $n$ nonlinear equations in the $m, r$ parameters of the gain matrix $K$ can be derived by setting the appropriate determinant in equation (24) equal to zero. However, no general conclusions can be drawn regarding the existence of the solution.

An alternative approach not involving solution of nonlinear equations is to assign $t$ eigenvalues as in equation (14) and to attempt to assign the remaining ( $n-t)$ eigenvalues approximately to the subsystem in equation (18) using the condition in equation (24). In this case, a set of ( $n-t)$ linear equations in $m$ unknowns results, and a least-squares solution can be obtained. The foregoing discussions assume that $r \geqq m$ if necessary by considering the dual system. 


\section{NUMERICAL EXAMPLES}

Example 1

Consider the system described in reference 7 where

$$
\left.\begin{array}{l}
\bar{A}=\left[\begin{array}{llll}
0 & 1 & 0 & 0 \\
0 & 0 & 0 & 0 \\
0 & 0 & 0 & 1 \\
0 & 0 & 0 & 0
\end{array}\right] \\
\bar{B}=\left[\begin{array}{ll}
0 & 0 \\
1 & 0 \\
0 & 0 \\
0 & 1
\end{array}\right] \\
\bar{C}=\left[\begin{array}{llll}
1 & 0 & 0 & 0 \\
0 & 0 & 1 & 0
\end{array}\right]
\end{array}\right\}
$$

Reduce the system (25) to the special form of equations (1) by ordering state variables as $\left(\mathrm{x}_{1}, \mathrm{x}_{3}, \mathrm{x}_{2}, \mathrm{x}_{4}\right)$ to make $\overline{\mathrm{C}}_{\mathrm{a}}$ nonsingular (appendix $A$ ) and by applying the coordinate transformation $(\overline{\mathrm{C}}, \overline{\mathrm{A}}, \overline{\mathrm{B}}) \rightarrow\left(\overline{\mathrm{CT}}_{0}, \mathrm{~T}_{0}{ }^{-1} \overline{\mathrm{A}}_{0}, \mathrm{~T}_{0}{ }^{-1} \overline{\mathrm{B}}\right)$ where

$$
\mathrm{T}_{0}=\left[\begin{array}{llll}
1 & 0 & 0 & 0 \\
0 & 1 & 1 & 1 \\
0 & 0 & 1 & 0 \\
0 & 0 & 0 & 1
\end{array}\right]
$$


to yield

$$
\begin{aligned}
& A=\left[\begin{array}{llll}
0 & 0 & 1 & 0 \\
0 & 0 & 0 & 1 \\
0 & 0 & 0 & 0 \\
0 & 0 & 0 & 0
\end{array}\right] \\
& B=\left[\begin{array}{lll}
0 & 0 \\
-1 & -1 \\
1 & 0 \\
0 & 1
\end{array}\right] \\
& C=\left[\begin{array}{llll}
1 & 0 & 0 & 0 \\
0 & 1 & 1 & 1
\end{array}\right]
\end{aligned}
$$

From equation (6) it can be shown that the closed-loop eigenvectors satisfy

$$
\left[\begin{array}{ll}
\lambda & 0 \\
0 & \lambda
\end{array}\right]\left[\begin{array}{l}
n_{2} \\
n_{1}
\end{array}\right]=\left[\begin{array}{cc}
-\lambda & (1-\lambda) \\
1 & 0
\end{array}\right]\left[\begin{array}{l}
n_{3} \\
n_{4}
\end{array}\right]
$$

where the eigenvalue is $\lambda$ and the eigenvector is

$$
\mathrm{v}=\left(\begin{array}{llll}
\mathrm{n}_{1} & \mathrm{n}_{2} & \mathrm{n}_{3} & \mathrm{n}_{4}
\end{array}\right)^{\prime}
$$

From theorem 2, condition I, $n_{1} \neq 0$; therefore, choose $n_{1}=1$ without loss of generality. Then, $\tilde{N}_{2}=\left(\begin{array}{lll}-n_{2} & -n_{3} & -n_{4}\end{array}\right)^{\prime}$. Condition II is met for all choices of $\widetilde{\mathrm{N}}_{2}$ since $\mathrm{B}_{1}^{\hat{1}}=0$. Condition III implies that

$$
\left\{\left[\begin{array}{ccc}
0 & 0 & 0 \\
-n_{2} & -n_{3} & -n_{4} \\
1 & 0 & 0
\end{array}\right],\left[\begin{array}{l}
1 \\
1 \\
1
\end{array}\right]\right\}
$$

should be controllable. For the controllability matrix in equation ( 30 ) to be of full rank, the eigenvector parameters in equation (28) must satisfy

$$
n_{2} n_{3}-n_{4}+n_{3}^{2}+n_{3} n_{4} \neq 0
$$

By direct substitution equation (31) is seen to be violated by all admissible selections in equation (28). Thus, the system cannot be assigned $(m+r-1)=3$ eigenvalues. Indeed, only two eigenvalues can be assigned to 
this system (ref. 7). However, the previous analyses (refs. 3 and 7) do not precisely lead to this conclusion.

Example 2

Consider the system described by (ref. 7)

$$
\left.\begin{array}{l}
\bar{A}=\left[\begin{array}{lll}
0 & 1 & 0 \\
0 & 0 & 1 \\
0 & 0 & 0
\end{array}\right] \\
\bar{B}=\left[\begin{array}{ll}
1 & 0 \\
1 & 0 \\
1 & 1
\end{array}\right] \\
\bar{C}=\left[\begin{array}{lll}
1 & 0 & 0 \\
0 & 1 & 0
\end{array}\right]
\end{array}\right\}
$$

It is required to assign eigenvalues close to $-1,-2$, and -5 . The system (32) can be reduced to the form of equations (1) by following the procedure in appen$\operatorname{dix} A$ as

Step 1: Measurement matrix is in the desired form with $\overline{\mathrm{C}}_{\mathrm{a}}$ nonsingular.

Step 2: Apply coordinate transformation

$$
(\overline{\mathrm{C}}, \overline{\mathrm{A}}, \overline{\mathrm{B}}) \rightarrow\left(\overline{\mathrm{C}} \mathrm{T}_{0}, \mathrm{~T}_{0}{ }^{-1} \overline{\mathrm{A}}_{0}, \mathrm{~T}_{0}{ }^{-1} \overline{\mathrm{B}}\right)
$$

where

$$
\mathrm{T}_{0}=\left[\begin{array}{lll}
1 & 0 & 0 \\
0 & 1 & 1 \\
0 & 0 & 1
\end{array}\right]
$$


yielding

$$
\left.\begin{array}{l}
A=\left[\begin{array}{lll}
0 & 1 & 1 \\
0 & 0 & 1 \\
0 & 0 & 0
\end{array}\right] \\
B=\left[\begin{array}{ll}
1 & 0 \\
0 & -1 \\
1 & 1
\end{array}\right] \\
C=\left[\begin{array}{lll}
1 & 0 & 0 \\
0 & 1 & 1
\end{array}\right]
\end{array}\right\}
$$

The system (34) is in the required canonical form of equations (1). The closedloop eigenvector constraints in equations (34) can be derived by identifying the respective matrices in equations (8) to (10) as

$$
\begin{aligned}
& B_{1}=\left[\begin{array}{ll}
1 & 0 \\
0 & -1
\end{array}\right] \\
& S=\left(\begin{array}{ll}
1 & -1
\end{array}\right) \\
& F=0 \\
& G=\left(\begin{array}{ll}
0 & -1
\end{array}\right)
\end{aligned}
$$

and equation (6) yields

$$
\lambda_{W}=\left[\begin{array}{ll}
\lambda & -(1+\lambda)
\end{array}\right]\left[\begin{array}{l}
z_{1} \\
\cdot \cdot \\
z_{2}
\end{array}\right]
$$

Equation (35) indicates that $z_{1}$ and $z_{2}$ can be arbitrarily chosen, provided $\lambda \neq 0$. Now, applying the algorithm to assign $(m+r-1)$ eigenvalues yields the following synthesis sequence.

\section{Step 1:}

Assign $\lambda=-1$. From appendix $B$, case II, $\Delta_{t}(1)=\{1\}$, and this implies $z_{1} \neq 0$ in equation (35). (Condition $I$, theorem 2.) One acceptable assignment is 


$$
N=\left(\begin{array}{lll}
1 & 1 & 1
\end{array}\right)
$$

and a nonunique feedback gain corresponding to this assignment from equation (B6) is

$$
\widetilde{K}=\left[\begin{array}{rr}
-1 & -1 \\
0 & 1
\end{array}\right]
$$

The closed-loop system matrix of equation (14) is

$$
\tilde{A}=\left[\begin{array}{ccc}
-1 & 0 & 0 \\
0 & -1 & 0 \\
-1 & 0 & 0
\end{array}\right]
$$

Step 2:

Transform the system to the canonical form of equations (16) using

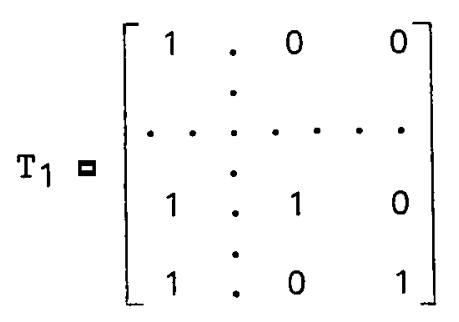

to yield

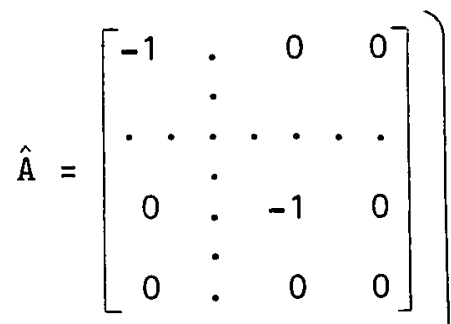

$$
\begin{aligned}
& \hat{B}=\left[\begin{array}{cc}
1 & 0 \\
\cdot & \cdot \\
-1 & -1 \\
0 & 1
\end{array}\right] \\
& \hat{\mathrm{C}}=\left[\begin{array}{llll}
1 & \cdot & 0 & 0 \\
2 & \dot{*} & 1 & 1
\end{array}\right]
\end{aligned}
$$


From equations (21) we can form the dynamic system

$$
\left.\begin{array}{l}
\dot{\xi}=\left[\begin{array}{ll}
0 & 0 \\
1 & 0
\end{array}\right] \xi+\left[\begin{array}{l}
1 \\
1
\end{array}\right] \mu_{1}+\left[\begin{array}{l}
1 \\
1
\end{array}\right] \mu_{2} \\
\eta=\left[\begin{array}{cc}
0 & 1 \\
-1 & 1
\end{array}\right] \xi+\left[\begin{array}{l}
1 \\
0
\end{array}\right] \mu_{1}
\end{array}\right\}
$$

with

$$
-\mu_{1}=\left(\begin{array}{ll}
1 & 1
\end{array}\right) \xi, \quad \mu_{2}=\left(\begin{array}{ll}
-1 & 1
\end{array}\right) \eta
$$

Since feedback from $\mu_{1}$ does not affect the controllability of system (41) with respect to $\mu_{2}$, the subsystem is pole assignable from condition III of theorem 2. Further, the assignment in step 1 satisfies condition II since $\hat{\mathrm{B}}_{2}$ is nonsingular. Thus theorem 2 holds.

\section{Step 3:}

Choose $\ell^{\prime}=\left(\begin{array}{ll}-2 & 1\end{array}\right)$. This choice protects $\lambda=-1$ assigned in step 1 . It now remains to choose $q$ so that $\hat{K}=q l$ ' assigns the eigenvalues $\lambda_{2}=-2$ and $\lambda_{3}=-5$.

Step 4: where

Assign $\lambda_{2}=-2, \lambda_{3}=-5$ to the single output subsystem in equation (18)

$$
\left.\begin{array}{l}
\hat{A}_{22}=\left[\begin{array}{cc}
-1 & 0 \\
0 & 0
\end{array}\right] \\
\hat{B}_{2}=\left[\begin{array}{cc}
-1 & -1 \\
0 & 1
\end{array}\right] \\
c=\left[\begin{array}{ll}
1 & 1
\end{array}\right]
\end{array}\right\}
$$


with feedback

$$
\begin{aligned}
& q=\left[\begin{array}{c}
6 \\
-10
\end{array}\right] \\
& \hat{\mathrm{K}}=\left[\begin{array}{c}
6 \\
-10
\end{array}\right]\left[\begin{array}{ll}
-2 & 1]
\end{array}\right\}
\end{aligned}
$$

Step 5:

The feedback law of the form of equation (22) is given by

$$
u=\left\{\left[\begin{array}{rr}
-1 & -1 \\
0 & 1
\end{array}\right]+\left[\begin{array}{rr}
-12 & 6 \\
20 & -10
\end{array}\right]\right\} \mathrm{y}
$$

and assigns the eigenvalues $-1,-2$, and -5 to the system (eq. (32)).

\section{AIRCRAFT LATERAL CONTROL DESIGN}

The advantage of combined control of closed-loop eigenvalues and eigenvectors using state variable feedback has been investigated in reference 10 . The utility of the output feedback extensions developed in this report will now be illustrated through the design of a lateral controller for an aircraft.

The linear perturbation model for the lateral motions of an aircraft can be modeled as

$$
\left.\begin{array}{l}
\dot{x}=\bar{A} x+\bar{B} u \\
y=\bar{C} x+\bar{D} u
\end{array}\right\}
$$

where $x$ is the state vector of roll rate $p$, yaw rate $\dot{\psi}$, sideslip $\beta$, and bank angle $\phi$, respectively. The control vector of aileron $\delta_{a}$ and rudder $\delta_{r}$ angular deflections is $u$. Roll rate $p$, yaw rate $\psi$, and lateral acceleration $a_{y}$ constitute the output vector $y$. All angles are in degrees, rates in $\mathrm{deg} / \mathrm{sec}$, and acceleration in $\mathrm{m} / \mathrm{sec}^{2}\left(\mathrm{ft} / \mathrm{sec}^{2}\right)$.

The respective matrices in equations (45) for a fighter aircraft at an altitude of $6096 \mathrm{~m}$ (20 $000 \mathrm{ft}$ ), a Mach number of 0.67 , and an angle of attack of $3.45^{\circ}$ are given by 


$$
\begin{aligned}
& \bar{A}=\left[\begin{array}{cccc}
-3.79 & 0.04 & -52 & 0 \\
-0.14 & -0.36 & 4.24 & 0 \\
0.06 & -1 & -0.27 & 0.05 \\
1 & 0.06 & 0 & 0
\end{array}\right] \\
& \bar{B}=\left[\begin{array}{cc}
25 & 9.83 \\
1.42 & -4.2 \\
0.01 & 0.05 \\
0 & 0
\end{array}\right] \\
& \overline{\mathrm{C}}=\left[\begin{array}{cccc}
1 & 0 & 0 & 0 \\
0 & 1 & 0 & 0 \\
-0.13 & -0.06 & -3.42 & 0
\end{array}\right] \\
& \widetilde{D}=\left[\begin{array}{cc}
0 & 0 \\
0 & 0 \\
1.03 & -0.27
\end{array}\right]
\end{aligned}
$$

where the elements of the matrices are approximated to two significant digits.

Since the output vector in equations (45) is derived as a linear combination of both state variables and control inputs, the closed-loop system after applying feedback $u=K^{*} y$ takes the form

$$
\left.\begin{array}{l}
x=(\bar{A}+\bar{B} K \bar{C}) x+\overline{B P u} \\
y=(\bar{C}+\bar{D} K \bar{C}) x+\bar{D} P u
\end{array}\right\}
$$

where $P=\left[I_{m}-K^{*} \bar{D}\right]^{-1}$ and $K=\mathrm{PK}^{*}$ is the equivalent output feedback matrix obtained by setting $\overline{\mathrm{D}}=0$. Thus, the algorithms developed earlier for systems with $\bar{D}=0$ are applicable to systems of the form of equations (45), provided $P$ exists.

Then the feedback gain $K^{*}$ is computed by using the relation

$$
\mathrm{K}^{*}=\mathrm{K}\left[\mathrm{I}_{\mathrm{k}}+\overline{\mathrm{DKK}}\right]-1
$$


Direct matrix manipulations show that the inverse in equation (47) exists if $P$ exists.

The handling qualities specifications (ref. 11) imply that the lateral aircraft dynamics should be composed of two weakly coupled subsystems. Roll rate and bank angle constitute the first subsystem and display predominantly the roll subsidence and spiral modes. The second subsystem is characterized by a welldamped Dutch roll mode defining the yaw rate and sideslip motions. These specifications can now be formulated as an eigenvalue/eigenvector assignment problem.

Table I summarizes the modal characteristics of the free aircraft. From the table it is seen that the Dutch roll mode is very lightly damped and appears dominantly in the response of the roll variables $p$ and $\phi$ as evidenced by dominant entries in the corresponding eigenvector pair. Thus, the eigenvalue/ eigenvector modification requires that the closed-loop system have the modes and mode-variable associations of table II. (In tables I to III, $j=\sqrt{-1}$.)

TABLE I.- MODAL CHARACTERISTICS OF FREE AIRCRAFT

\begin{tabular}{|c|c|c|c|c|}
\hline \multirow{2}{*}{$\begin{array}{l}\text { Eigenvector } \\
\text { components }\end{array}$} & \multicolumn{4}{|c|}{ Eigenvalue of - } \\
\hline & $\begin{array}{ll} & -3.70 \\
\text { (Roll subsidence) }\end{array}$ & $\begin{array}{l}-0.35 \pm \\
\text { (Dutch }\end{array}$ & $\begin{array}{l}j 2.66 \\
11)\end{array}$ & $\begin{array}{l}-0.03 \\
\text { (Spiral) }\end{array}$ \\
\hline $\mathrm{p}$ & -0.964 & -0.403 & 0.829 & -0.032 \\
\hline$\dot{\psi}$ & -.041 & -.096 & -.131 & .044 \\
\hline$\beta$ & -.002 & .069 & -.034 & .002 \\
\hline$\phi$ & .261 & .324 & .112 & .998 \\
\hline
\end{tabular}

TABLE II.- DESIRED MODAL SPECIFICATIONS

Mode

Roll subsidence

Dutch roll

Spiral
Eigenvalue $-6$

$-1.0 \pm j 0.2$

$-0.01$
Dominant response variable

Roll rate

Yaw rate and sideslip

Bank angle 
The output feedback analysis in the main text shows that all system eigenvalues can be assigned since $n=m+r-1$ and only two eigenvectors ( $t$ ) can be assigned with at most two $(m)$ entries in each vector arbitrarily chosen. The eigenvector freedom available was used to control the structure of the eigenvector pair corresponding to the Dutch roll mode to effect the desired yaw rate and sideslip dominance. The modal coupling matrices $\mathrm{D}(\mathrm{k})$ (eq. (B4)) aid in the selection of the Dutch roll mode to yield the appropriate eigenvector forms. The four eigenvector entries that were freely chosen corresponded to the roll rate and yaw rate components of the real eigenvector pair associated with the Dutch roll mode. Since gain magnitude constraints cannot be explicitly included into the synthesis algorithm, the design parameters have to be iteratively modified to meet gain limit requirements. After some design iterations, a compromise design yielded the modal characteristics summarized in table III. For example, it was noted that the Dutch roll mode damping could not be reduced (to improve $\dot{\psi}$ and $\beta$ responses) without violating feedback gain limits which were set at unity for this analysis. The design yielded a feedback gain matrix $K^{*}$ (eq. (47)) as

$$
K^{*}=\left[\begin{array}{lll}
-0.19 & -0.2 & -0.66 \\
-0.16 & 0.19 & -0.6
\end{array}\right]
$$

TABLE III.- MODAL CHARACTERISTICS OF FEEDBACK AUGMENTED AIRCRAFT

\begin{tabular}{|c|c|c|c|c|}
\hline \multirow{2}{*}{$\begin{array}{l}\text { Eigenvector } \\
\text { components }\end{array}$} & \multicolumn{4}{|c|}{ Eigenvalue of - } \\
\hline & $\begin{array}{c}-6 \\
\text { (Roll subsidence) }\end{array}$ & $\begin{array}{l}-1.0 \\
\text { (Dutc }\end{array}$ & $\begin{array}{l}j 0.20 \\
\operatorname{rol} 1)\end{array}$ & $\begin{array}{c}-0.011 \\
\text { (Spiral) }\end{array}$ \\
\hline $\mathrm{p}$ & 0.986 & 0.01 & 0.02 & -0.013 \\
\hline$\dot{\psi}$ & .009 & .35 & .53 & -.031 \\
\hline$B$ & -.007 & .25 & .72 & -.082 \\
\hline$\phi$ & -.165 & -.05 & .05 & -.996 \\
\hline
\end{tabular}

Table III illustrates that the closed-loop eigenvectors have approached the desired mode-decoupled structure. In particular, the desired modification achieved in the eigenvector pair corresponding to the Dutch roll mode should be noted. The improvement in transient response characteristics using the feedback controller is illustrated in figure 1. The response curves demonstrate that the cross coupling between the roll axis $(p, \phi)$ and yaw axis $(\psi, \beta)$ has been significantly reduced. 


\section{CONCLUDING REMARKS}

New sufficient conditions to assign minimum $(n, m+r-1)$ eigenvalues by means of output feedback have been derived. In general, in addition to the assignment of minimum $(n, m+r-1)$ eigenvalues, $(r-1)$ eigenvectors can be partially assigned with at most $m$ entries in each vector arbitrarily chosen. The utility of assigning both eigenvalues and eigenvectors for response modification is illustrated by designing a feedback controller for the lateral dynamics of an aircraft. The synthesis algorithm is computationally simple and involves only the solution of a linear system of equations.

\section{Langley Research Center}

National Aeronautics and Space Administration

Hampton, VA 23665

December 19, 1977 
Consider the given system of the form

$$
\left.\begin{array}{l}
\dot{\bar{x}}=\bar{A} \bar{x}+\bar{B} u \\
y=\bar{C} \bar{x}
\end{array}\right\}
$$

Step 1:

Reorder the state variables (if necessary) so that the measurement matrix is of the form

$$
\overline{\mathrm{C}} \cdot\left[\overline{\mathrm{C}}_{\mathrm{a}}: \overline{\mathrm{C}}_{\mathrm{b}}\right]
$$

where $\bar{C}_{a} \in R^{r \times r}$ and nonsingular. It should be noted that the dimensions of the partitioned matrices in equation (A2) are different from those in equations ( 3 ).

Step 2:

Apply a coordinate transformation

$$
\bar{x}=T_{0} x
$$

where

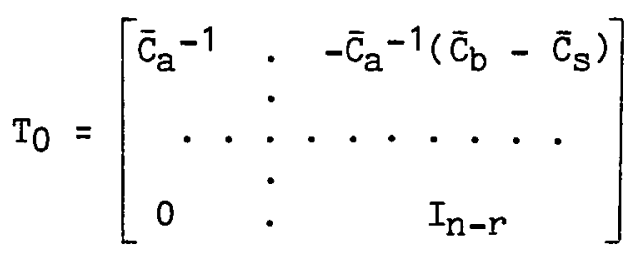

and $\overline{\mathrm{C}}_{\mathrm{S}} \in \mathrm{R}^{\mathrm{r} \times \mathrm{n}-\mathrm{r}}$ and $\overline{\mathrm{C}}_{\mathrm{S}}=\left[\begin{array}{c}0 \\ \cdot \\ \mathrm{c}\end{array}\right]$ with $\mathrm{c}$ as defined in equations (3). The transformed system

$$
\left.\begin{array}{l}
\dot{\mathrm{x}}=\left(\mathrm{T}_{0}{ }^{-1} \overline{\mathrm{A}} \mathrm{T}_{0}\right) \mathrm{x}+\mathrm{T}_{0}{ }^{-1} \overline{\mathrm{Bu}} \\
\mathrm{y}=\overline{\mathrm{C}} \mathrm{T}_{0} \mathrm{x}
\end{array}\right\}
$$

has the desired form of equations (1). 


\section{APPENDIX B}

\section{EIGENVALUE/EIGENVECTOR SELECTION PROCEDURE}

For completeness of presentation, an existing algorithm (ref. 8) developed for eigenvalue/eigenvector assignment using state variable feedback is detailed in this appendix. Extensions are made to adopt the algorithm for the output feedback cases discussed in the main text.

\section{SPECTRAL SYNTHESIS ALGORITHM}

A direct way of constructing a nonsingular modal matrix is to generate the eigenvectors which satisfy equation (6) sequentially and insure that they do not lie in the eigenspace generated by the vectors already synthesized. The algorithm presented accomplishes this construction while constantly testing to insure that the set of eigenvectors is a linearly independent set to a degree determined by a numerical tolerance parameter set by the designer. For clarity of presentation the algorithm is detailed for real eigenvalue assignments. The following notations are used throughout the algorithm presentation: equal to zero.

(1) $e_{j}$ is an n-vector with jth entry equal to 1 and all other entries

(2) $v_{k}^{\prime}$ a $\left[z_{k}^{\prime}: w_{k}^{\prime}\right]$ is the $k t h$ eigenvector, where $z_{k}$ is designer specified.

(3) $v_{k}(k-1)=Q^{(k-1)} V_{k}$, where $Q^{(0)}=I_{n}$ and $Q^{(i)}$, i $\neq 0$, is defined in (7) which follows.

(4) $v_{j k}(k-1)$ is the jth entry of $v_{k}(k-1)$ and for convenience of notation is denoted $\sigma_{\mathrm{k}}$.

(5) $\Delta(1)$ is the set of indices $(1,2, \ldots, \ldots, n)$.

(6) $\mathrm{M}(\mathrm{k})$, an elementary upper triangular matrix of order $\mathrm{n}$ and index 1 , takes the form

$$
M^{(k)}=I_{n}-\theta e_{j}^{\prime}
$$

with $e_{j}^{\prime} \theta=0$ and $\theta$ is chosen so that

$$
M^{(k)} V_{k}(k-1)=\sigma_{k} e_{j}, j \in\{\Delta(k)\}
$$

where $\Delta(k)$ is a subset of $\Delta(1)$ (defined in (5)) containing the indices not already used in the construction of the matrices $M^{(1)}, M^{(2)}, . . ., M(k-1)$. $\mathrm{M}^{(\mathrm{k})}$ can be constructed if and only if $\sigma_{k} \neq 0$ (ref. 8).

(7) $\mathrm{Q}^{(\mathrm{k}-1)}=\mathrm{M}^{(\mathrm{k}-1)}, \mathrm{M}^{(\mathrm{k}-2)}, . ., \mathrm{M}^{(1)}$ 


\section{APPENDIX B}

The algorithm is now accomplished by completing steps 1 to 4 for $\mathrm{k}=1,2, \ldots ., \mathrm{n}$ and by computing the feedback gains from equation (12) using $C=I_{n}$.

\section{Step 1:}

For $\lambda=\lambda_{\mathrm{k}}$, compute $\delta_{\mathrm{k}}=\operatorname{det}\left[\lambda_{\mathrm{k}} I_{\mathrm{n}-\mathrm{m}}-\mathrm{F}\right]$ $\delta_{\mathrm{k}}$

(a) if $\delta_{k}=0$, perturb $\lambda_{k}$ to $\left[\lambda_{k}+\varepsilon \lambda_{k}\right]$ and repeat the calculation of

(b) if $\delta_{k} \neq 0$, proceed to step 2 .

Step 2:

Compute

$$
D^{(k)}=\left[\lambda_{k} I_{n-m}-F\right]^{-1}\left[G+\lambda_{k} S\right]
$$

Step 3:

For some $j \in\{\Delta(k)\}$,

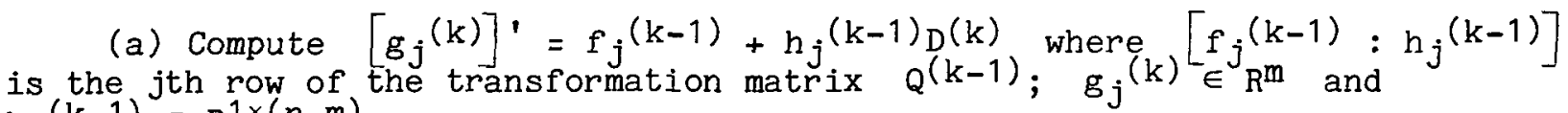
$h_{j}(k-1) \in R^{1 \times(n-m)}$.

(b) Compute

$$
\sigma_{k}=\left[g_{j}(k)\right]^{\prime} z_{k}
$$

(i) if $\sigma_{k} \neq 0$, compute $w=D^{(k)} z_{k}$ and $M^{(k)}$ (from eq. (B2)) and return to step 1 for the next value of $k$.

(ii) if $\sigma_{k}=0$, select another $j \in\{\Delta(k)\}$ and return to step $3(a)$.

$$
\text { (iii) if } \sigma_{k}=0 \text { for all } j \in\{\Delta(k)\} \text {, go to step } 4 \text {. }
$$

Step 4:

For some $j \in\{\Delta(\mathrm{k})\}$,

(a) if $g_{j}(k) \neq 0$, perturb $z_{k}$ to $\left(z_{k}+\varepsilon z_{k}\right)$ to make $\sigma_{k} \neq 0$, compute $w_{k}$ and $M(k)$, and return to step 1 for the next value of $k$.

(b) if $g_{j}(k)=0$, select another $j \in\{\Delta(k)\}$ and return to step $4(a)$.

(c) if $g_{j}(k)=0$ for all $j \in\{\Delta(k)\}$, perturb $\lambda_{k}$ to $\left(\lambda_{k}+\varepsilon \lambda_{k}\right)$ and return to step 1 . 
The following observations can be made regarding the algorithm as outlined.

(i) Extension of the algorithm to assign complex eigenvalues is straightforward (ref. 8).

(ii) If some of the eigenvalues of $\Lambda$ coincide with those of $F$, either a perturbation can be made in the eigenvalue specification as in step 1 , or special eigenvector structures can be derived by noting that equation (6) has a solution for $z_{i}=0$ (ref. 8 ).

(iii) The iterative procedure in part ii of step 3(b) attempts to meet exact eigenvalue/eigenvector specifications. In step $4(\mathrm{~b})$ an attempt is made to meet exact $\lambda_{k}$ specifications with slightly relaxed $z_{k}$ specifications. The test in step $4(\mathrm{c})$ indicates that the $\lambda_{\mathrm{k}}$ specification implied that the corresponding $v_{k}$ lies in the eigenspace already generated. The test thus demands an eigenvalue perturbation.

(iv) The $\sigma_{k}$ provide a good measure of the linear independence between eigenvectors, if all vectors are normalized to a standard basis. Since the determinant of the modal matrix $\mathrm{V}$ is given by the product of the $\sigma_{k}$, ( $k=1,2, \ldots . ., n$ ), the numerical ill conditioning of $\mathrm{V}$ for inversion can be effectively controlled by setting a tolerance on the $\sigma_{k}$.

\section{OUTPUT FEEDBACK EXTENSIONS}

Since the distribution matrix $\mathrm{C}$ is already in the special canonical form, generating linearly independent vectors to guarantee existence of feedback matrices in equations (12) and (14) is simply achieved by restricting the admissible pivotal indices $\Delta(\mathrm{k})$ (eq. (B2)) in the spectral synthesis algorithm as follows.

\section{Case I: Computing $\overline{\mathrm{K}}$, Equation (12)}

In equation (5), let $\Delta_{r}(1)$ be the set of $r$ column indices corresponding to the $r$ linearly independent columns of $C\left(I_{r}\right)$ (i) Then apply the spectral synthesis algorithm using $\Delta_{r}(1)$ instead of $\Delta(i)$ (notation (5)) for $\mathrm{k} \cdot 1,2, \cdot . ., \mathrm{r}$. This application guarantees the invertibility of $\mathrm{CV}(\mathrm{r})$ in equation (12).

\section{Case II: Computing $\tilde{\mathrm{K}}$, Equation (14)}

In equation (5), let $\Delta_{t}(1)$ be the set of $t$ column indices not containing the vector $c$ (eqs. (3)). Then apply the spectral synthesis algorithm using $\Delta_{t}(1)$ instead of $\Delta(1)$ (notation (5)) for $k=1,2, . . ., t$. This application guarantees the nonsingularity of $\mathrm{N}_{1}$ (eq. (13)). Further, since in this case $C N$ is rank $t$, the feedback matrix $\widetilde{K}$ (eq. (14)), computed using the relation

$$
\tilde{\mathrm{K}} \mathrm{CN}=\mathrm{B}_{1}-1\left[\mathrm{Z}^{(\mathrm{t})} \Lambda_{\mathrm{t}}-\mathrm{A}_{1} \mathrm{~N}\right]
$$


to assign $t$ eigenvalues, is not unique. However, a minimum norm least-squares solution for $\widetilde{\mathrm{K}}$ can be computed using the pseudo inverse of $\mathrm{CN}$. 


\section{REFERENCES}

1. Wonham, W. M.: On Pole Assignment in Multi-Input Controllable Linear Systems. IEEE Trans. Autom. Control, vol. AC-12, no. 6, Dec. 1967, pp. 660-665.

2. Srinathkumar, S.; and Rhoten, R. P.: Eigenvalue/Eigenvector Assignment for Multivariable Systems. Electronics Lett., vol. 11, no. 6, Mar. 1975, pp. 124-125.

3. Kimura, Hidenori: Pole Assignment by Gain Output Feedback. IEEE Trans. Autom. Control, vol. AC-20, no. 4, Aug. 1975, pp. 509-516.

4. Kimura, Hidenori: A Further Result on the Problem of Pole Assignment by Output Feedback. IEEE Trans. Autom. Control, vol. AC-22, no. 3, June 1977, pp. 458-463.

5. Hermann, Robert; and Martin, Clyde F.: Applications of Algebraic Geometry to Systems Theory - Part I. IEEE Trans. Autom. Control, vol. AC-22, no. 1, Feb. 1977, pp. 19-25.

6. Topaloglu, Toros; and Seborg, Dale E.: An Algorithm for Pole Assignment Using Output Feedback. Proceedings: 1974: Joint Automatic Control Conference, American Inst. Chem. Eng., c.1974, pp. 309-312.

7. Davison, E. J.; and Wang, S. H.: On Pole Assignment in Linear Multivariable Systems Using Output Feedback. IEEE Trans. Autom. Control, vol. AC-20, no. 4, Aug. 1975, pp. 516-518.

8. Srinathkumar, S.: Spectral Characterization of Multi-Input Dynamic Systems. Ph. D. Diss., Oklahoma State Univ., 1976.

9. Davison, E. J.; and Chatterjee, R.: A Note on Pole Assignment in Linear Systems With Incomplete State Feedback. IEEE Trans. Autom. Control, vol. AC-16, no. 1, Feb. 1971, pp. 98-99.

10. Srinathkumar, S.; and Rhoten, R. P.: Eigenvalue/Eigenvector Control Via Spectral Characterization: An Application to Helicopter Hover Dynamics. Ninth Annual Asilomar Conference on Circuits, Systems, and Computers, Shu-Park Chan, ed., Western Periodical Co., (N. Hollywood, Calif.), Nov. 1975, pp. 605-609.

11. Hartmann, Gary L.; Hauge, James A.; and Hendrick, Russell C.: F-8C Digital CCV Flight Control Laws. NASA CR-2629, 1976. 

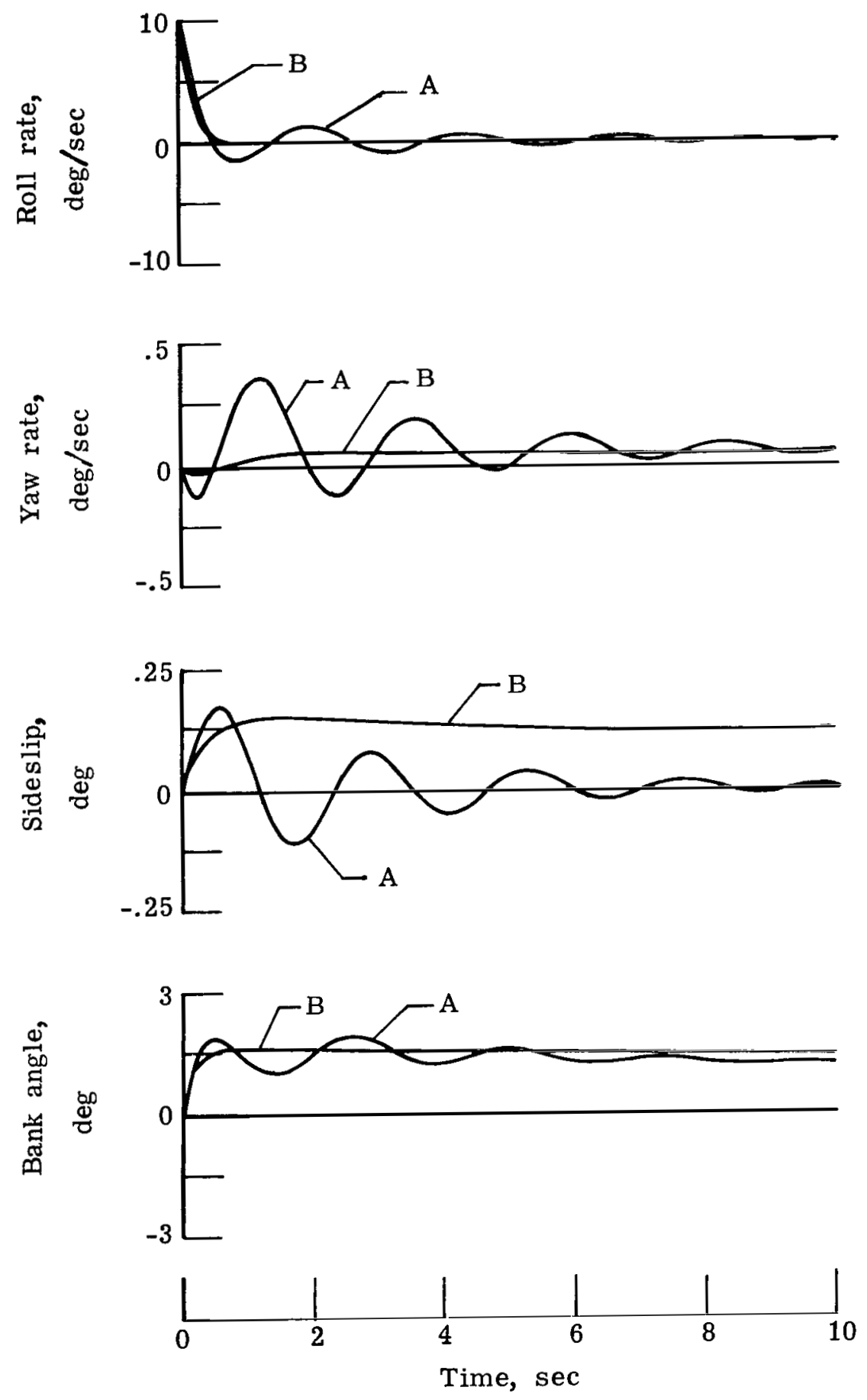

(a) $p(0)=10 \mathrm{deg} / \mathrm{sec}$.

Figure 1.- Comparison of free aircraft and augmented aircraft response to roll rate and sideslip step disturbances. A indicates free aircraft response and $B$ corresponds to augmented aircraft response. 

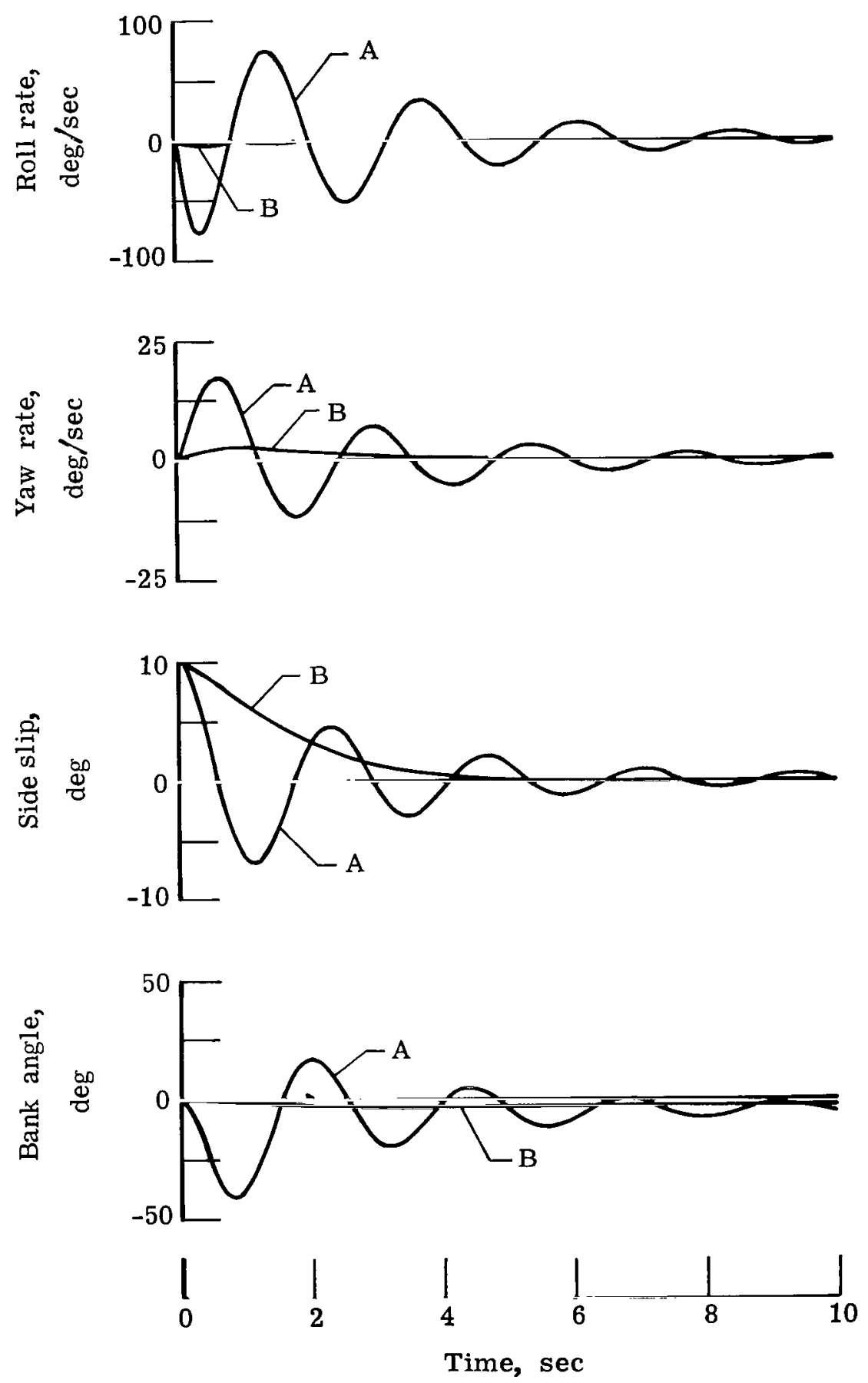

(b) $B(0)=10^{\circ}$.

Figure 1.- Concluded. 


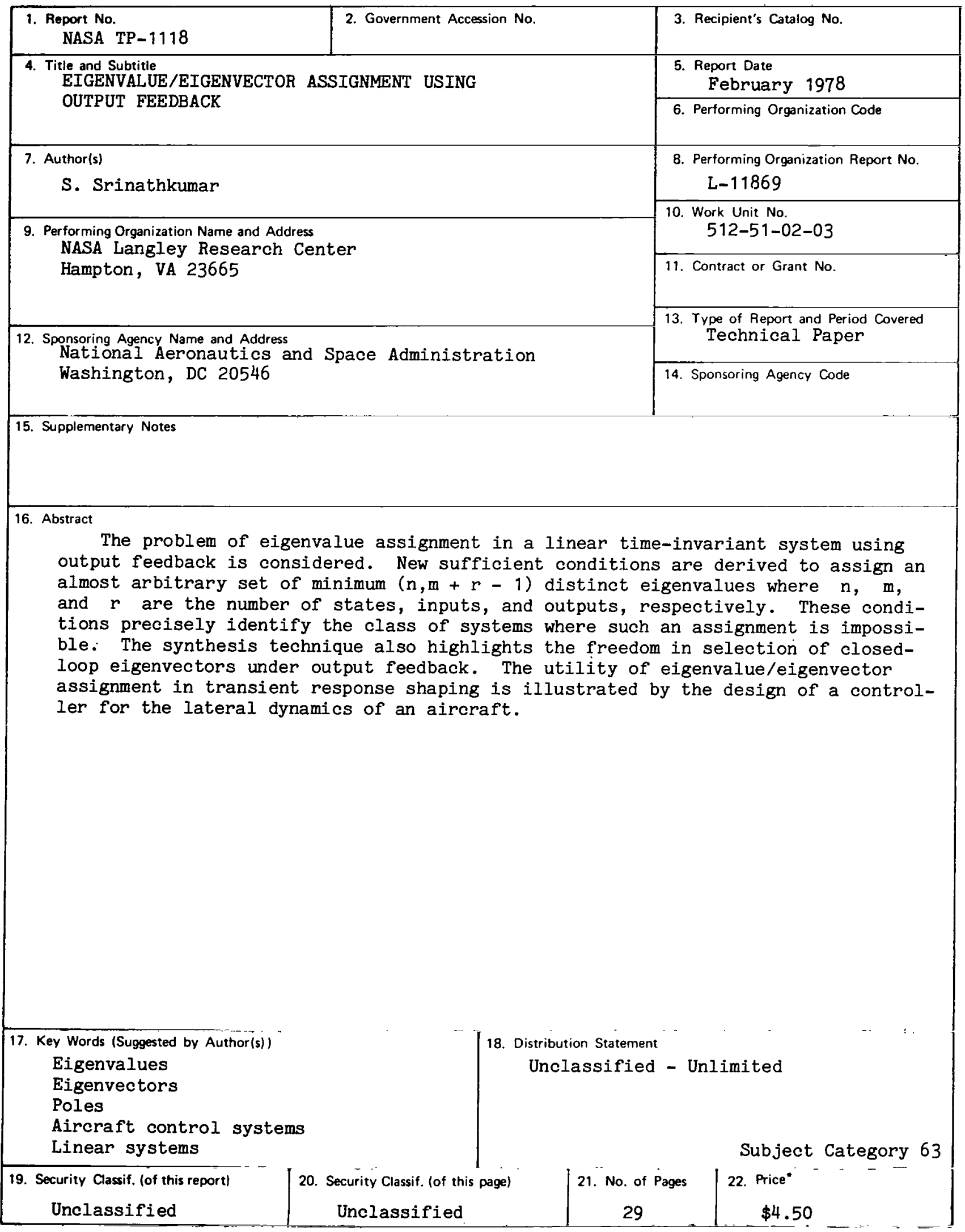

* For sale by the National Technical Information Service, Springfield, Vırginia 22161 
National Aeronautics and

Space Administration

Washington, D.C.

20546

Official Business

Penalty for Private Use, $\$ 300$
Postage and Fees Paid National Aeronautics and Space Administration NASA-451

012378 SOU9J315

$15110, \mathrm{G}$,

DEPT OF THE AIR FORCE

AF WEAEONS LAEORATORY

ATTN: TECHNICAL IIBRARY (SUL)

KIRTLAND AFB NM 87117

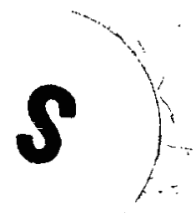

